\title{
Contamination during production of heater-cooler units by Mycobacterium chimaera potential cause for invasive cardiovascular infections: results of an outbreak investigation in Germany, April 2015 to February 2016
}

S Haller ${ }^{1}$, C Höller ${ }^{2}$, A Jacobshagen ${ }^{3}$, O Hamouda $^{1}$, M Abu Sin ${ }^{1}$, DL Monnet ${ }^{4}$, D Plachouras ${ }^{4}$, T Eckmanns ${ }^{1}$

1. Department for Infectious Disease Epidemiology, Robert Koch Institute (RKI), Berlin, Germany

2. Bavarian Health and Food Safety Authority (LGL), Oberschleißheim, Germany

3. Federal Institute for Drugs and Medical Devices (BfArM), Bonn, Germany

4. European Centre for Disease Prevention and Control (ECDC), Stockholm, Sweden

Correspondence: Tim Eckmanns (eckmannst@rki.de)

Citation style for this article:

Haller S, Höller C, Jacobshagen A, Hamouda O, Abu Sin M, Monnet DL, Plachouras D, Eckmanns T. Contamination during production of heater-cooler units by Mycobacterium chimaera potential cause for invasive cardiovascular infections: results of an outbreak investigation in Germany, April 2015 to February 2016 . Euro Surveill. 2016;21(17): pii=30215. DOI: http://dx.doi.org/10.2807/1560-7917.ES.2016.21.17.30215

Article submitted on 23 March 2016 / accepted on 18 April 2016 / published on 28 April 2016

Invasive infections with Mycobacterium chimaera were reported in patients with previous open chest surgery and exposure to contaminated heater-cooler units (HCUs). We present results of the surveillance of clinical cases and of contaminated HCUs as well as environmental investigations in Germany up until February 2016. Clinical infections occurred in five male German cases over 50 years of age (range $53-80$ ). Cases had been exposed to HCUs from one single manufacturer during open chest surgery up to five years prior to onset of symptoms. During environmental investigations, $M$. chimaera was detected in samples from used HCUs from three different countries and samples from new HCUs as well as in the environment at the manufacturing site of one manufacturer in Germany. Our investigation suggests that at least some of the $M$. chimaera infections may have been caused by contamination of HCUs at manufacturing site. We recommend that until sustainable measures for safe use of HCUs in operation theatres are implemented, users continue to adhere to instructions for use of HCUs and Field Safety Notices issued by the manufacturer, implement local monitoring for bacterial contamination and continuously check the websites of national and European authorities for current recommendations for the safe operation of HCUs.

\section{Introduction}

In July 2014, the Federal Office of Public Health Switzerland (FOPH) reported about patients with Mycobacterium chimaera infections, who had previously undergone open-chest heart surgery with exposure to contaminated heater-cooler units (HCUs) [1]. Several other reports and publications have suggested since that HCUs produced by one manufacturer in
Germany may be a source of $M$. chimaera infections that occurred in Switzerland, Germany, the Netherlands and United Kingdom [2-5].

HCUs are commonly used in cardiac surgery during extracorporeal circulation in order to regulate the temperature of the blood and to provide temperature-controlled water for cardioplegia. HCUs have water tanks that provide temperature-controlled water to external heat exchangers. Since $M$. chimaera was detected in air samples close to operating HCUs, airborne transmission is believed to be the most likely transmission mechanism in the $M$. chimaera cases after open chest surgery $[4,6]$.

M. chimaera is a slow-growing nontuberculous mycobacterium (NTM) belonging to the $M$. avium complex (MAC). It was first reported by Tortoli et al. in 2004 as a closely to $M$. intracellulare-related distinct species [7]. Identification requires molecular diagnostic testing [8]. M. chimaera may cause lung infections especially in patients with underlying lung disease as well as disseminated infections in immunocompromised patients and was found in skin and bone infections. In the environment, it was identified in biofilms and detected in water sources such as household water [9].

Among others, the report by the FOPH about the outbreak investigations in Switzerland and the reports about cases in Germany and the Netherlands led to increased surveillance efforts and outbreak investigations in Europe $[3,10]$. Here we present the results of the surveillance of clinical cases, of the surveillance of contaminated HCUs and of environmental investigations in Germany. 
Cases with symptomatic Mycobacterium chimaera infection, notified between April 2015 and February 2016, Germany $(\mathrm{n}=5)$

\begin{tabular}{|l|c|c|c|c|c|c|c|c|}
\hline $\begin{array}{l}\text { Case } \\
\text { number }\end{array}$ & $\begin{array}{c}\text { Age } \\
\text { (years) }\end{array}$ & Sex & $\begin{array}{c}\text { Cardiac } \\
\text { surgery } \\
\text { centre }\end{array}$ & $\begin{array}{c}\text { Type of surgery } \\
\text { (exposure) }\end{array}$ & $\begin{array}{c}\text { Prosthetic } \\
\text { material }\end{array}$ & Site of infection & $\begin{array}{c}\text { Death } \\
\text { due to } \\
\text { infection }\end{array}$ & $\begin{array}{c}\text { Incubation } \\
\text { period } \\
\text { (years) }\end{array}$ \\
\hline 1 & 80 & Male & A & Aortic valve replacement & Yes & Endocarditis & No & \multicolumn{1}{|c|}{ (1 } \\
\hline 2 & 75 & Male & B & CABG & No & Spondylodiscitis & No & 5 \\
\hline 3 & 65 & Male & C & Aortic valve replacement & Yes & $\begin{array}{c}\text { Valvular aortic endocarditis, } \\
\text { paravalvular leak and } \\
\text { abscess }\end{array}$ & Yes & 3 \\
\hline 5 & 67 & Male & C & $\begin{array}{c}\text { CABG and aortic valve } \\
\text { replacement }\end{array}$ & Yes & Paravalvular abscess ${ }^{\text {c }}$ & No $^{\text {b }}$ & 4 \\
\hline 5 & 53 & Male & C & Aortic valve replacement & Yes & $\begin{array}{c}\text { Endocarditis and cerebral } \\
\text { abscesses }\end{array}$ & No & 3 \\
\hline
\end{tabular}

CABG: coronary artery bypass grafting.

a Time between exposure to open chest surgery involving use of an HCU and clinical diagnosis.

${ }^{\mathrm{b}}$ Currently in palliative care.

' Endocarditis lenta and change of aortic valve in September 2013.

\section{Methods}

\section{Definitions}

For our investigations we used the following case definitions: a confirmed case was defined as a patient having undergone surgery with extracorporeal circulation in the five years before onset of symptoms of NTM infection AND in whom M. chimaera was detected in an invasive sample (e.g. blood, tissue biopsy or implanted prosthetic material). A probable case was defined as a confirmed case, but without detection of $M$. chimaera in an invasive sample.

An HCU was considered as contaminated, when cardiac surgery centres found NTM and/or other bacteria from environmental samples from the HCU and sent a report to the Federal Institute for Drugs and Medical Devices (BfArM) in Germany.

\section{Prospective case finding and identification of contaminated HCUs \\ Prospective case finding was conducted from April 2015 onwards and results until end February 2016 are presented here. The mandatory surveillance of health- care-associated outbreaks in Germany was applied for reporting clinical cases and this surveillance is described in detail elsewhere [11].}

The public health authorities and healthcare professionals in Germany were informed about the ongoing outbreak and requested to notify cases fulfilling the case definition [12]. Specifically, the German National public health institute (Robert Koch Institute (RKI)), the German Society of Thoracic and Cardiovascular Surgery and the German Society of Infection informed federal states' authorities and societies' members, respectively, about case definitions and notification according to the article 6 of the 'Protection against Infection Act' (Infektionsschutzgesetz, IfSG) [12-15].

The mandatory notification system for incident reports of medical devices was used to detect contaminated HCUs in Germany. Incident reports were collected and analysed by BfArM in accordance with the corresponding legal framework 'The Act on Medical Devices' (Medizinproduktegesetz) and 'The Medical Device Safety Plan' (Medizinprodukte Sicherheitsplanverordnung).

HCU users were requested to submit any incident report associated with HCUs to BfArM [16]. On 10 July 2015, the BfArM recommended to place HCUs outside of the operation theatre and monitoring of contamination in HCUs [17].

At the European level, the European Centre for Disease Prevention and Control (ECDC) assessed the risk of invasive cardiovascular infection by $M$. chimaera potentially associated with heater-cooler units used during cardiac surgery in Europe also, in April 2015 [10]. The risk assessment was forwarded to regional German public health authorities. From April 2015 onwards, ECDC also provided a platform for exchange of information and a protocol for case detection and environmental testing [18]. The protocol was shared with all European Union/European Economic Area (EU/EEA) countries with the purpose to obtain information in a harmonised way, to further investigate the association between invasive infection by $M$. chimaera and HCUs, and to allow assessing the burden of these infections. The protocol was shared with the German heart surgery centres that detected clinical cases. 
Mycobacterium chimaera-positive samples from environmental investigations at the manufacturing site of new HCUs and of used HCUs from at the manufacturer's service centre, July 2014 to June 2015

\begin{tabular}{|l|l|c|}
\hline Date & Type of sample & Source of sample \\
\hline 16 Jul 2014 & Water $(100 \mathrm{~mL})$ & Used HCU from Switzerland \\
\hline 29 Jul 2014 & Water $(100 \mathrm{~mL})$ & New HCU from manufacturing site \\
\hline 5 Aug 2014 & Water $(100 \mathrm{~mL})$ & New HCU from manufacturing site \\
\hline 11 Aug 2014 & Water $(100 \mathrm{~mL})$ & Used HCU from the Netherlands \\
\hline 19 Feb 2015 & Water $(100 \mathrm{~mL})$ & Sample taken in pump assembly area at the manufacturing site \\
\hline 10 Jun 2015 & Water (volume not specified) & \\
\hline
\end{tabular}

HCU: heater-cooler unit.

The environmental investigations were performed by the manufacturer.

Investigation at the HCU manufacturing site and at the manufacturers' service centre In July 2015, the Bavarian Health and Food Safety Authority (LGL), assisted the Bavarian regulatory authorities with on-site investigations and took environmental samples at the manufacturing site and in the service centre of the implicated manufacturer. Samples were taken from the production line, on-site tap water and from a used and disassembled HCU from this manufacturer in the service centre. All samples were sent to the National Reference Centre (NRC) for Mycobacteria Borstel, Germany.

On its own initiative, the HCU manufacturer conducted environmental sampling for NTM at the manufacturing site where the HCUs are assembled and in the service centre where used HCUs are disassembled for decontamination from July 2014 onwards. Environmental samples were sent to a local microbiological laboratory and NTM isolates were submitted to the NRC in Borstel for further analysis.

\section{Culturing and typing}

Mycobacteria were cultured in different laboratories. The development of standard protocols for microbiological M. chimaera diagnostic was coordinated by ECDC in collaboration with laboratories such as the NRC Borstel in Europe; these protocols were published by ECDC in August 2015 [18].

Next generation sequencing (NGS) of isolates is still ongoing.

\section{Ethics}

A formal ethical review process and approval was not required for this outbreak investigation in accordance with article 25 , section 1 of the IfSG.

\section{Results}

At the beginning of our investigation, in April 2015, we were informed by cardiac surgery centre A in Germany about a confirmed case that became symptomatic before 2015 [3]. During April 2015 to February 2016, the mandatory surveillance of healthcare-associated outbreaks identified four additional confirmed cases of $M$. chimaera infection who had been exposed to an $\mathrm{HCU}$ in two different cardiac surgery centres (B and C) in Germany (Table 1). These cases developed a symptomatic $M$. chimaera infection five months to five years after exposure to a HCU. All five confirmed cases were male and aged above 50 years (range 53-80) when diagnosed with $M$. chimaera infection, four had aortic valve replacement and two underwent coronary artery bypass grafting, one died. All had been exposed to HCUs from one single manufacturer during open chest surgery. No cases with NTM infections other than $M$. chimaera were notified. Our investigations did not reveal epidemiological links between cases of the different sites.

Between January 2015 and February 2016, the BfArM received 26 incident reports of contaminated HCUs from 16 of the total of 78 German cardiac surgery centres from different German regions. Three of the 16 centres reported contamination of HCUs of another manufacturer but $M$. chimaera detection from these HCUs was not reported. Overall, the contaminations of the HCUs included $M$. chimaera and other bacteria such as Pseudomonas aeruginosa, Legionella pneumophila and Stenotrophomonas maltophilia and fungi. All three centres in which German cases were exposed sent incident reports about contamination of HCUs from the same German manufacturer. Two of these centres reported $M$. chimaera detection in HCU water samples including one reporting also detection of $M$. chimaera in air samples. The third centre reported NTM in HCU water samples, results of further specification were not reported.

During the environmental investigations performed by the Bavarian regulatory authorities on 2 July 2015, six of 20 samples obtained were $M$. chimaera-positive. All positive samples were from one disassembled HCU that had been used in cardiac surgery centre $D$ in Germany and was disassembled for decontamination in the service centre of the manufacturer. The disassembled HCU was produced before modifications in the post-production process that were implemented 
by the manufacturer in response to the findings of $M$. chimaera contamination. The samples included in the investigations were water (ca $100 \mathrm{~mL}$ ), swab and biofilm and were collected from different sources: residual water, filler neck, patient bridge, biofilm from patient recirculation and patient bath.

In December 2015, the HCU manufacturer provided the RKI with information about six $M$. chimaera-positive samples from environmental investigations conducted between July 2014 and June 2015, including two contaminated HCUs from Switzerland and the Netherlands, respectively (Table 2 ).

On 22 December 2015, public health authorities in the EU/EEA and worldwide were notified by Germany about the suspected common source of $M$. chimaera via the EU Early Warning and Response System (EWRS) and via an International Health Regulation (IHR) notification.

\section{Discussion}

We present data that show that $M$. chimaera was isolated in clinical samples from (i) infected patients in Germany who had undergone open chest surgery, (ii) in samples from used HCUs from three different countries and (iii) in samples from new HCUs and the environment at the manufacturing site of one manufacturer. This suggests that at least some of the five German cases with $M$. chimaera infection may have occurred due to contamination of the HCUs by $M$. chimaera at the manufacturing site.

Preliminary typing results indicate that the $M$. chimaera isolates detected by the authorities and the isolates from the manufacturer appear to be almost identical (unpublished data). The M. chimaera-positive environmental samples at the manufacturing site prompted the manufacturer to modify the manufacturing process, which now includes ethanol disinfection and an active drying of the HCU water circuit before shipment. When the Bavarian regulatory authorities conducted onsite visits, no $M$. chimaera-positive sample was recovered except from a used HCU which had been disassembled for decontamination. The returned unit had been manufactured before August 2014. According to the information provided by the manufacturer, HCUs manufactured before mid-August 2014 may have had environmental mycobacteria presence in the unit at the time of delivery. Our investigations could not elucidate if and until when contaminated HCUs may have been delivered to customers from this manufacturer.

As of end of March 2016, two additional notifications of patients with $M$. chimaera-positive clinical specimens are under investigation in Germany. Until now we could not obtain data on all surgical interventions prior the M. chimaera diagnosis of these patients.

A limitation of our study is that we did not conduct active case finding. It is likely that the passive surveillance has led to an underestimation of the actual number of cases of $M$. chimaera infections in Germany. Furthermore, the true number of cases is probably underestimated since there is no typical clinical picture for infections with $M$. chimaera. Patients present with nonspecific symptoms, a variety of infection sites and a culture for mycobacteria is usually not part of a routine diagnostic work-up in patients presenting with signs of infection.

M. chimaera was not the only bacterial species isolated from HCUs. Contamination of HCUs with other bacteria was reported from various cardiac surgery centres in Germany. Furthermore, bacteria were also isolated from HCUs produced by other manufacturers. It is possible that some of the cases were infected due to contamination of HCUs at the cardiac surgery centres. It is also possible that some of the cases occurred due to exposure to HCUs produced by other manufacturers.

Infections by $M$. chimaera are rare and their occurrence, when detected, is considered unusual [19]. The reported $M$. chimaera infections might therefore be regarded as an indicator of a potential microbial hazard caused by the water-bearing HCUs in the healthcare environment.

Further investigations are needed to differentiate between the risk of $M$. chimaera infection from HCUs contaminated at the manufacturing site, the risk of infection from HCUs contaminated during use and the risk of infection from other medical devices that include an HCU such as extracorporeal membrane oxygenators [20]. In two recent publications, Götting et al. and Sommerstein et al. gave interesting insights into possible mechanisms of airborne transmission by HCUs $[4,6]$. In the cases described here, NGS should help determine the fraction that may be due to contamination at the manufacturing site or during use at the cardiac surgery centres.

To allow for targeted public health action, it is important that manufacturers of medical products share the findings of their own investigations into bacterial contamination, as demonstrated in this outbreak investigation. Sharing the results by the manufacturer, as well as information on the implemented corrective measures, allowed us to better understand the risks involved in HCU use. Regulatory authorities in Germany are continuing their information exchange with the manufacturers that produce HCUs to provide a sustainable solution for minimising the risks of infection in patients exposed to HCUs.

\section{Conclusions}

We present evidence on $M$. chimaera detection in clinical samples from infected German patients having been exposed to HCUs produced by the same manufacturer, in three cardiac surgery centres, in samples from used HCUs from three different countries and in samples from new HCUs and the environment at the manufacturing site of one manufacturer. In summary, 
this suggests a point source for the reported $M$. chimaera infections and for $M$. chimaera-positive samples from HCUs and the environment. Notifications of contaminated HCUs of different manufacturers and with various bacteria, indicate a general problem with water-bearing systems in the healthcare environment.

We recommend that until sustainable measures for a safe use of HCUs in operation theatres are implemented, users continue to adhere to the instructions for use of the HCU and the Field Safety Notices issued by the manufacturer, implement a local monitoring for bacterial contamination of the HCUs and continuously check the websites of relevant national and European authorities for current recommendations for the safe operation of HCUs.

\section{Acknowledgements}

We would like to thank the HCU manufacturer for sharing their investigation results with the German authorities. The authors would like to thank colleagues of the NRC Borstel for the microbiological analysis. Furthermore, we would like to thank health personnel as well as local and regional public health authorities who notified clinical cases and contaminated HCUs. Finally we would like to thank HP Blank who supported surveillance and data management at RKI.

\section{Conflict of interest}

The authors have shared the manuscript with the manufacturer before publication. This has not led to changes of the content. The authors have declared that they have no competing interests.

\section{Authors' contributions}

$\mathrm{SH}, \mathrm{MAS}, \mathrm{OH}$ and TE were part of the outbreak team at RKI and conducted the epidemiological outbreak investigations. $\mathrm{SH}, \mathrm{TE}$ and DP designed the investigation. SH, TE, DP, AJ, DLM and CH drafted the manuscript. All authors critically revised the manuscript and approved the final version. TE is corresponding author and guarantor.

\section{References}

1. Federal Office of Public Health Switzerland (FOPH). Massnahmen für höhere Patientensicherheit in der Herzchirurgie. [Measures for improved patient safety in cardiac surgery]. Press release. FOPH. Updated 14 Jul 2014. Available from: https://www.news.admin.ch/message/index. html?lang=de\&msg-id $=53774$

2. Sax H, Bloemberg G, Hasse B, Sommerstein R, Kohler P, Achermann Y, et al. Prolonged Outbreak of Mycobacterium chimaera Infection After Open-Chest Heart Surgery. Clin Infect Dis. 2015;61(1):67-75. DOI: 10.1093/cid/civ198 PMID: 25761866

3. Kohler P, Kuster SP, Bloemberg G, Schulthess B, Frank M, Tanner FC, et al. Healthcare-associated prosthetic heart valve, aortic vascular graft, and disseminated Mycobacterium chimaera infections subsequent to open heart surgery. Eur Heart J. 2015;36(40):2745-53. DOI: 10.1093/eurheartj/ehv342 PMID: 26188001

4. Sommerstein R, Rüegg C, Kohler P, Bloemberg G, Kuster SP, Sax H. Transmission of Mycobacterium chimaera from heatercooler units during cardiac surgery despite an ultraclean air ventilation system.Emerg Infect Dis. 2016;22(6). DOI: 10.3201/ eid2206.160045 PMID: 27070958

5. Public Health England (PHE). Mycobacterial infections associated with cardiopulmonary bypass surgery, 10 June 2015. London: PHE. Available from: https://www.gov.uk/ government/news/mycobacterial-infections-associated-withcardiopulmonary-bypass-surgery

6. Götting T, Klassen S, Jonas D, Benk C, Serr A, Wagner D, et al. Heater-cooler units: contamination of crucial devices in cardiothoracic surgery. J Hosp Infect. 2016 Feb 27. pii: S01956701(16)00126-2. doi: DOI: 10.1016/j.jhin.2016.02.006 . [Epub ahead of print]

7. Tortoli E, Rindi L, Garcia MJ, Chiaradonna P, Dei R, Garzelli C, et al. Proposal to elevate the genetic variant MAC-A, included in the Mycobacterium avium complex, to species rank as Mycobacterium chimaera sp. nov. Int J Syst Evol Microbiol. 2004;54(Pt 4):1277-85. DOI: 10.1099/ijs.0.02777-o PMID: 15280303

8. Schweickert B, Goldenberg O, Richter E, Göbel UB, Petrich $A$, Buchholz $P$, et al. Occurrence and clinical relevance of Mycobacterium chimaera sp. nov., Germany. Emerg Infect Dis. 2008;14(9):1443-6. DOI: 10.3201/eid1409.071032 PMID: 18760016

9. Wallace RJ, lakhiaeva E, Williams MD, Brown-Elliott BA, Vasireddy S, Vasireddy R, et al. Absence of Mycobacterium intracellulare and presence of Mycobacterium chimaera in household water and biofilm samples of patients in the United States with Mycobacterium avium complex respiratory disease. J Clin Microbiol. 2013;51(6):1747-52. DOI: 10.1128/JCM.00186-13 PMID: 23536397

10. European Centre for Disease Prevention and Control (ECDC). Invasive cardiovascular infection by Mycobacterium chimaera potentially associated with heater-cooler units used during cardiac surgery. Stockholm: ECDC. 30 Apr 2015. Available from: http://ecdc.europa.eu/en/publications/Publications/ mycobacterium-chimaera-infection-associated-with-heatercooler-units-rapid-risk-assessment-30-April-2015.pdf

11. Haller S, Eckmanns T, Benzler J, Tolksdorf K, Claus H, Gilsdorf $A$, et al. Results from the first 12 months of the national surveillance of healthcare associated outbreaks in Germany, 2011/2012. PLoS One. 2014;9(5):e98100. DOI: 10.1371/journal. pone.0098100 PMID: 24875674

12. Robert-Koch-Institut (RKI). Informationen zu einem internationalen Ausbruchsgeschehen mit nichttuberkulösen Mykobakterien im Zusammenhang mit Termperaturregulierungsgeräten ber Herzoperationen. [Information on an international outbreak with nontuberculous Mycobacteria connected to heater cooler units]. Epidemiologisches Bulletin. 2015;20:165. German. Available from: https://www.rki.de/DE/Content/Infekt/EpidBull/ Archiv/2015/Ausgaben/20_15.pdf?__blob=publicationFile

13. Deutsche Gesellschaft für Thorax- Herz- und Gefäßchirurgie (DGTHG) / Deutsche Gesellschaft für Infektiologie (DGI). Wichtige Information der DGTHG und DGI zu Infektionen mit Mycobacterium chimaera nach Herz-Operationen. [Important information of German Society of Thoracic and Cardiovascular Surgery and the German Society of Infection about Mycobacterium chimaera infections occurring after heart surgery]. Coswig: DGTHG/DGI. 30 Apr 2014. German. Available from: www.dgfkt.de/content/hygiene/information_DGTHGDGI_zu_m_chimaera_2015_04_30.pdf

14. Robert Koch Institute (RKI). Informationen zu einem internationalen Ausbruchsgeschehen mit nichttuberkulösen Mykobakterien im Zusammenhang mit Temperaturregulierungsgeräten bei Herzoperationen. [Information on an international outbreak with non-tuberculous Mycobacteria connected to heater cooler units]. Berlin: RKI. 2015. Available from: https://www.rki.de/DE/Content/ Infekt/Krankenhaushygiene/Aufb_MedProd/Mycobacterium_ chimaera.html

15. Mohr O, Velasco E, Fell G, Burckhardt F, Poggensee G, Eckmanns T. [Teleconferences for national surveillance of infectious diseases and public health events in Germany. Evaluation after three quarters in 2009]. Bundesgesundheitsblatt Gesundheitsforschung Gesundheitsschutz. 2010;53(9):903-9. DOI: 10.1007/s00103010-1122-z PMID: 20853087

16. Federal Institute for Drugs and Medical Devices (BfArM). (Mandatory notification for incident reports of medical devices in case of Mycobacterium chimaera infection following heart surgery). Medizinprodukte-Beobachtungs- und Medlesystem bei Infektionen mit Myobacterium chimaerea nach HerzOperationen beachten.Hyg Med. 2015;7/8(40):232.

17. Federal Institute for Drugs and Medical Devices (BfArM). Möglicher Zusammenhang zwischen Hypothermiegeräten und Infektionsrisiko mit Mykobakterien bei der Herzchirurgie. [Possible association between heater-cooler units and risk for mycobacterial infections in heart surgery]. Bonn: Germany. Updated 10 Jul 2015. Available from: http://www.bfarm. de/SharedDocs/Risikoinformationen/Medizinprodukte/DE/ Hypothermiegeraete.htm.

18. European Centre for Disease Prevention and Control (ECDC). EU protocol for case detection, laboratory diagnosis and 
environmental testing of Mycobacterium chimaera infections potentially associated with heater cooler units: case definition and environmental testing methodology. Stockholm: ECDC. Aug 2015. Available from: http://ecdc.europa.eu/en/publications/ Publications/EU-protocol-for-M-chimaera.pdf

19. Yuan SM. Mycobacterial endocarditis: a comprehensive review. Rev Bras Cir Cardiovasc. 2015;30(1):93-103.PMID: 25859873

20. Kanamori H, Weber DJ, Rutala WA. Healthcare-Associated

Outbreaks Associated with a Water Reservoir and Infection Prevention Strategies.Clin Infect Dis. 1 Mar 2016. [Epub ahead of print]

\section{License and copyright}

This is an open-access article distributed under the terms of the Creative Commons Attribution (CC BY 4.0) Licence. You may share and adapt the material, but must give appropriate credit to the source, provide a link to the licence, and indicate if changes were made.

This article is copyright of the authors, 2016. 